COMPTES RENDUS

\title{
IDÉES ET POLITIQUE
}

\author{
I Congressi degli scienziati italiani nell'età del positivismo. A cura di Giuliano \\ PanCaldi. Bologne, C.L.U.E.B., 1983. 15,3 x 21,3, 228 p.
}

Les neuf Congressi degli Scienziati Italiani qui eurent lieu entre 1839 et 1847 occupent une position importante dans l'historiographie du long processus qui aboutit, à partir de 1859 , à l'unification de l'Italie. Dans ce contexte, le rôle des Congressi est habituellement comparé à celui de la Deutscher Naturforscher Versammlung, qui s'embarqua dans ses pérégrinations à travers les États allemands en 1822. Cette interprétation est au fond plausible. Il n'est, en effet, pas permis de douter qu'en Italie comme en Allemagne ces réunions de savants servaient à rehausser le sentiment d'une identité nationale sur le plan culturel, à une époque où la bourgeoisie cultivée trouvait l'action politique infructueuse ou difficile. En effet, la biographie collective entreprise dans ce volume par Fernando Minuz et Annamaria Tagliavini tend à renforcer l'analogie entre l'évolution du sentiment nationaliste dans les deux pays. Selon leur étude, les Congressi auraient rassemblé un cercle assez cohérent d'universitaires et de membres des professions libérales sortis des universités, et ce fut ce même cercle qui marcha en tête du mouvement pour l'unification, surtout à partir du congrès de 1846 , tenu à Naples. Cette dernière date est importante, car ce ne fut qu'en 1846 que le groupe dirigeant, centré au départ sur la Lombardie et la région de Venise, réussit à s'attacher de nombreux collaborateurs des régions moins propices, notamment des États Pontificaux et du Royaume des Deux-Siciles.

Cela dit, il est évident qu'un historique des Congressi qui ne prend en considération que leurs aspects politiques et sociaux reste imparfait. Ainsi, un des intérêts essentiels des six articles et des deux appendices qui composent cet ouvrage est de dépasser ces points de vue, en attirant l'attention sur le contenu scientifique des débats des congressistes : à cette fin, les mathématiques, la géologie, là nomenclature zoologique, et les applications de la science sont traitées en détail. Il n'en reste pas moins qu'il n'est guère fait mention ni de la médecine ni de la chimie, d'où il suit, comme Giuliano Pancaldi le souligne dans son introduction, que le volume ne constitue qu'un premier élément de l'évaluation globale qu'il reste à faire. 
Il va sans dire que, pour l'historien, il serait à la fois satisfaisant et élégant que les débats scientifiques des Congressi se présentassent, à la suite de ces études, en quelque sorte comme le reflet des aspirations politiques des tenants de l'unité nationale. Mais ma lecture de l'ouvrage me laisse persuadé que cet accord du style cognitif et du contexte plus large des Congressi n'a pas été identifié, et que l'existence même d'un tel accord reste encore douteuse. Selon l'article d'Umberto Bottazzini, par exemple, les contributions des mathématiciens manifestaient une approche sans véritable cohérence ; dans ce domaine du moins, il serait difficile de croire que les Congressi favorisaient un style caractéristique. Peut-être ne pouvait-il en être autrement, étant donné les différences culturelles qui séparaient les divers États italiens et la structure dispersée de la communauté scientifique. Dans sa biographie récente d'Amedeo Avogadro, Mario Morselli démontre l'influence qu'exerçait cette structure sur la vie d'un savant piémontais qui cherchait à se faire connaître dans le premier quart du XIX $\mathrm{X}^{\mathrm{e}}$ siècle ; ces articles prouvent que la situation ne s'était guère améliorée dans les années 1840. Il est intéressant de noter, à ce sujet, que dans un commentaire (en grande partie favorable) sur sa participation au congrès de Turin de 1840, le physicien suisse, Auguste De La Rive, présentait encore la dispersion de l'activité scientifique en Italie comme un des aspects les plus frappants de la vie intellectuelle du pays.

Les mathématiques n'étaient assurément pas la seule discipline où régnait la diversité. Telle est la conclusion qui ressort incontestablement de la contribution de Nicoletta Morello sur la géologie. L'article de Pancaldi met également en évidence les obstacles qui empêchaient l'établissement d'une nomenclature uniforme en zoologie (tout en évoquant, débats de la British Association for the Advancement of Science à l'appui, les difficultés auxquelles étaient confrontés les partisans d'une telle nomenclature dans d'autres pays). Par contre, à la différence de ces domaines scientifiques, les applications de la science auraient été l'objet d'une uniformité beaucoup plus marquée. Comme le démontre Gian Carlo Calgano, l'amélioration des machines industrielles et des techniques agricoles était un but commun à de nombreux Italiens tout en servant simultanément de point de ralliement important à ceux qui servaient la cause nationaliste. Parmi les partisans principaux de l'unification, dont une très forte proportion était issue de la classe des industriels et des propriétaires, l'amélioration matérielle était considérée non seulement comme une réaction à un simple besoin économique mais aussi comme le symbole d'une activité qui dépassait les intérêts d'une région particulière et concernait toute la Péninsule.

Malgré les efforts du professeur Pancaldi et de ses collaborateurs, les lacunes et les incertitudes qui caractérisent l'historiographie des Congressi nous empêchent de situer avec certitude cette innovation spécifiquement italienne dans le contexte plus large de la science européenne. Dans quelle mesure, par exemple, sommes-nous fondés à assimiler les Congressi au mouvement général qui mena les communautés scientifiques de l'Allemagne, de la Grande-Bretagne et de la France à lancer leurs congrès itinérants dans les années 1820 et 1830 ? La qualité des communications des Congressi justifierait-elle cette assimilation? Certainement - et c'est tout ce qu'on peut dire 
pour le moment - il y a lieu de croire que bien des savants étrangers voyaient dans les Congressi un rassemblement de talents scientifiques tout à fait comparable à ceux des congrès qui existaient déjà dans d'autres pays d'Europe. C'est ce qui explique le vif intérêt que portaient, à cette initiative, des participants du niveau de De La Rive, de Carl Gustav Jacobi, et de Charles Babbage. Sur le plan purement italien, d'ailleurs, il serait difficile de douter de l'importance intellectuelle des Congressi alors que parmi leurs adhérents figuraient les noms d'Ottaviano Fabrizio Mossotti, Carlo Matteucci, Giovanni Plana, et Giovanni Battista Amici.

Ces preuves, à la fois de la qualité supérieure des Congressi et de la totale reconnaissance de celle-ci, même à l'étranger, provoquent une dernière réflexion d'ordre historiographique. On accepte, en effet, sans difficulté que la cause nationaliste en Italie souffrît de l'interruption des Congressi à cause des événements politiques de 1848 (les congrès ne devaient reprendre qu'après 1870). Mais les événements de 1848 ne provoquèrent-ils pas une autre perte à plus long terme, celle de la visibilité de la science italienne au nord des Alpes ? On est tenté de croire, d'après la lecture de ce volume, que si les Congressi avaient pu poursuivre leurs activités dans les années 1850 et 1860 , la Péninsule ne serait pas devenue cette terra incognita telle qu'elle risque d'apparaître, tout à fait à tort, dans les perspectives des historiens non italiens de la science européenne du XIX ${ }^{e}$ siècle.

Robert Fox.

Jules Ferry, fondateur de la République. Actes du colloque organisé par l'École des hautes études en sciences sociales, présentés par François FURET. Paris, E.H.E.S.S., 1985. $16 \times 24,256$ p. ("Civilisations et sociétés », 72).

Dans sa présentation, F. Furet a précisé les objectifs du colloque qui s'est tenu en 1982 : étudier la vie, la formation, les idées et l'action d'un des grands dirigeants républicains et, par-là, approfondir nombre de questions cruciales pour notre connaissance de l'évolution de l'histoire politique et intellectuelle de la France à l'apogée de la IIIe République. L'ouvrage est riche de nombreuses communications regroupées autour de quelques grands thèmes, mais nous regrettons qu'il n'ait donné aucun écho aux discussions qui ont animé le colloque, fût-ce sous la forme d'une présentation résumée et synthétique des principaux points abordés.

L'étude de l'homme privé dessine un personnage issu d'un milieu de juristes de province et profondément atteint par les conséquences du coup d'État qui l'écarta de la fonction publique, objet de ses ambitions. Donc, dès l'abord, une destinée politique qui le conduit à s'insérer dans le groupe des républicains s'opposant au Second Empire, avec une adhésion précoce aux doctrines positivistes, ce qui, plus tard, devait lui donner des soutiens non négligeables. Plus 
anecdotiques, les apports consacrés à l' " homme intime ", à la place que Jules Ferry assignait à la femme dans la société sont révélateurs d'une sensibilité réelle mais cachée. Comme de nombreux représentants de l'élite bourgeoise de son temps, J. Ferry trouva auprès de son épouse réconfort, inspiration et conseil, mais, dans la tradition de Michelet et des positivistes, il érigea une situation de fait en système, il donna à la femme un rôle éminent et en fit une pierre angulaire de la société républicaine, sans pour autant lui donner les droits du citoyen.

Les divers aspects de l'œuvre de l'homme public sont passés en revue. Député des Vosges, Ferry s'intéressait à sa circonscription et prêtait l'oreille aux doléances de ses électeurs, mais sans jamais sacrifier aux intérêts locaux ce qu'il devait à sa dignité et à ses fonctions d'homme politique national. On trouve dans cette analyse une bonne illustration du principe selon lequel, sous la IIIe République, un député se devait de refuser tout mandat impératif de ses commettants. Se décidant en son âme et conscience dans la conduite de sa vie politique, un républicain comme Ferry avait une démarche intellectuelle et morale proche de celle des grands notables de son temps qui se situaient parmi les conservateurs libéraux.

La plupart des domaines où l'action de Ferry laissa des traces durables sont passés en revue dans cet ouvrage. L'cuvre scolaire et la défense de la laïcité n'impliquaient pas une hostilité systématique à l'égard du catholicisme. Ferry voulait seulement que l'Église abandonnât ses prétentions au contrôle des consciences et des âmes, ce qui était pour lui une garantie de la liberté, un moyen d'assurer la dignité humaine puisqu'il croyait profondément à l'existence d'une morale naturelle, indépendante de tout dogme et de toute organisation sociale. Réaction positiviste sans doute, comme le souligne le texte, réaction où nous voyons aussi un exemple de la bonne conscience et de l'orgueil qui sont un des traits caractéristiques de la pensée bourgeoise du $\mathrm{XIX}^{\mathrm{e}}$ siècle français. Plusieurs contributions montrent avec pertinence que le patriotisme a constitué la ligne directrice de la politique extérieure et de la politique coloniale de J. Ferry qui, sans que rien ne l'y ait préparé, joua en ce domaine un rôle décisif. Son objectif était d'accroitre la grandeur et le prestige de la France dans le monde. Tout en jouant la carte de la détente avec l'Allemagne, il ne s'est pas laissé duper par Bismarck, son habileté manœuvrière lui ayant permis de mener une action coloniale, en redonnant aussi à la France " un des premiers rôles sur la scène européenne ». Autant et parfois plus que ceux qui l'ont attaqué, il répondait ainsi aux aspirations profondes des élites françaises et d'une partie importante de la population.

Les deux dernières contributions replacent l'œuvre de J. Ferry dans une longue continuité. E. Weber évoque l'évolution qui a abouti à transformer en citoyens français des paysans dont l'horizon était auparavant limité à un terroir, $\mathbf{R}$. Girardet souligne en revanche que les institutions républicaines mises progressivement en place ont permis aux anciennes aspirations du tiers-état de 1789 de s'imposer dans le pays. Deux approches différentes qui conduisent à une même constatation. Jules Ferry et les fondateurs de la III' République avaient foi dans le progrès humain et la démocratie qui, à leurs yeux, étaient inséparables de la diffusion de la morale et des connaissances de base indis- 
pensables pour que l'on pût donner à tous la plénitude des droits du citoyen. Les notions de droit et de devoir, de capacité et de citoyenneté étaient étroitement liées. La IIIe République était et se voulait démocratique, mais l'esprit de ses institutions et de son fonctionnement se situait dans la droite ligne des valeurs bourgeoises. Peut-être est-ce une lacune de ce colloque que de n'avoir pas évoqué, sauf par allusions, les liens entre l'action de Jules Ferry et la civilisation bourgeoise. Il reste que cet ouvrage est d'un grand intérêt par ce qu'il apporte et par ce qu'il suggère.

Adeline Daumard.

Sylviane LEPRUN, Le Théâtre des colonies : scénographie, acteurs et discours de l'imaginaire dans les expositions, 1855-1937. Paris, L'Harmattan, 1986. $16 \times 24,308$ p., pl., ill.

En un temps où l'épopée coloniale ne semble plus assombrir les consciences des Français, Le Théâtre des colonies tente de nous restituer les procédés de communication qu'utilisèrent les ancêtres de nos média pour construire un spectacle colonial cohérent à travers les moments forts qu'ont représentés les expositions.

Au carrefour de l'architecture et de l'ethnologie, enseignante à l'École d'architecture de Paris-La Villette, Sylviane Leprun se propose de faire revivre "la patiente construction scénographique que les expositions cristallisent". De l'Exposition universelle de 1867 à celle, internationale, de 1937, se joue en effet une pièce de théâtre aux pôles d'attraction immuables, l'aventure et l'exotisme. Pourtant, des palais de l'Algérie aux rues de Djenné, des villages sénégalais aux portes monumentales de style soudanais, le spectacle s'épuise. " En ces premiers temps, écrit l'auteur, [l'Exposition] apparut novatrice, habitée par un souci de vérité. En fin de parcours, elle accusait sa pédagogie, on ne voyait que son aspect muséographique " (p. 215).

En 1937, l'architecte en chef, J. Greber, décidait de s'écarter du « style d'exposition ", d'abandonner les palais monumentaux et le décor de "surcharge" pour donner désormais au visiteur-contribuable une «leçon de choses " sans trop d'art ni de procédés car " il sait ce que l'argent de chaque colonie lui coûte ". Ainsi s'appauvrissait la scénographie coloniale et se desséchait l'imaginaire. L'élection des Reines d'outre-mer dans le cadre de l'île des Cygnes remplaçait la danse du ventre des cafés maures. Le Parisien énamouré des mystérieuses filles du soleil applaudissait désormais les postulantes au titre envié de Miss France d'outre-mer, des étudiantes, des secrétaires ou des sténo-dactylos qui retourneraient sagement à leurs occupations après cet intermède. Mais si 1937 marque la fin des expositions coloniales, les représentations qu'elles sous-tendaient perdurent. À Montréal, en 1967, au pavillon de la 
Tunisie, il y avait toujours un café maure où un personnel en costume régional servait des spécialités locales. Une interprétation contemporaine d'un thème ancien.

Etait-ce une façon d'accepter à la fois le présent et le passé, comme le suggère l'auteur, une manière de dédramatiser l'histoire ? Ce serait prêter aux architectes des États libéraux une singulière distanciation.

Jacques MARSEILle.

Wolfgang Mommsen, Max Weber et la politique allemande : 1890-1920. Trad. de l'allemand. Paris, P.U.F., 1985. $15 \times 22,547$ p. (« Sociologies »).

Quiconque sait à quel point la publication en français d'études autres qu'anglo-saxonnes, fût-ce avec un quart de siècle de retard comme c'est le cas en l'occurrence, constitue encore un véritable exploit, ne peut que se réjouir de la parution de ce livre qui est déjà presque un classique outre-Rhin. Car cet ouvrage apporte, en dépit des maladresses de traduction, notamment de certains concepts wébériens, une vue d'ensemble de l'itinéraire politique de celui qui ne fut pas seulement l'un des pères de la sociologie que l'on connaît en France mais aussi un "idéologue " - plutôt qu'un homme politique - de la société allemande à un tournant capital de son histoire.

Dans la Préface à l'édition française de la $2^{e}$ édition revue et augmentée de son livre (1974), Wolfgang Mommsen, héritier, avec son frère jumeau Hans, de la vocation familiale d'historien, qu'illustra notamment le grand-père Theodor Mommsen, spécialiste éminent de l'Antiquité, qui dirigea d'ailleurs la thèse de Max Weber sur L'Histoire agraire romaine et sa signification pour le droit public et privé (1891), rappelle que son étude suscita " certains remous " lors de la première publication en R.F.A., en 1959. Les contemporains de W. Mommsen, qui avaient vécu l'agonie de la République de Weimar, puis le triomphe et l'effondrement du III' Reich et finalement la " rééducation " par les Alliés après 1945, croyaient, en effet, avoir trouvé en Max Weber le père spirituel de la démocratie libérale dont se réclamait la jeune République fédérale d'Allemagne. Croyance du reste partagée par l'auteur lorsqu'il projeta de retracer l'itinéraire politique de celui-ci, mais qui ne tarde pas à être démentie par l'analyse systématique des sources - souvent inédites (correspondance, articles, discours).

Traité de " dilettante" par l'establishment universitaire allemand de son temps, pour avoir abordé les disciplines les plus diverses allant de l'histoire économique et sociale à celle des religions en passant par la sociologie, le droit et les sciences politiques, Max Weber apparaît dans ses écrits non pas comme un pionnier de la démocratie libérale, mais comme un rénovateur du libéralisme national bismarckien. D'où la controverse passionnée des années 
soixante relayée, depuis peu en R.F.A., par celle de " la voie particulière " de l'Allemagne moderne et de sa bourgeoisie'.

Fil conducteur des dix chapitres de Mommsen, qui suivent dans ses moindres contradictions l'évolution de la pensée politique de Max Weber jusqu'à sa mort prématurée, à 56 ans, en 1920 : la conviction, pour ne pas dire l'obsession, du déclin de l'Allemagne dans la compétition entre grandes puissances du monde capitaliste moderne. Déclin qui ne peut être enrayé, selon Weber, qu'en procédant à une transformation radicale de ses structures étatiques et des mentalités, jugées archaïques, d'une bourgeoisie appelée à prendre en main les destinées économiques et politiques. À l'opposé du nationalisme sentimental de la plupart de ses concitoyens, Weber invoque "les lois de l'histoire " pour préconiser une « politique froide et brutale de grande puissance " au service d'intérêts culturels spécifiques. Surestimation et transfiguration, note W. Mommsen (p. 97), de l'idée de puissance à travers le concept de nation, qui "fut le propre de l'époque de l'impérialisme et conduisit finalement la vieille Europe à la catastrophe ". De l'appel à une politique allemande de grande puissance, mélange de discours fichtéen à la Nation allemande, d'apologie nietzschéenne du surhomme et de darwinisme social, lancé en 1895 , lors de sa conférence inaugurale à l'Université de Fribourg-en-Brisgau (comment ne pas songer à l'appel du recteur Heidegger dans la même université en 1933 ?) à l'exposé de janvier 1919, c'est-à-dire au lendemain de la défaite et de l'effondrement de l'empire, devant les étudiants libéraux de Munich ${ }^{2}$, s'exprime la même ardeur passionnée à renouer avec la « Realpolitik " du chancelier de fer auquel il reproche simplement d'avoir, par son hostilité à l'institution parlementaire, empêché la sélection d'une relève de chefs politiques. Car, et c'est sans doute l'aspect essentiel que Mommsen développe dans la partie centrale du livre (chap. 6) et sur lequel il revient longuement dans les deux derniers chapitres : la démocratisation parlementaire que Weber appelle inlassablement de ses vœux en invoquant l'efficacité du modèle anglais, diffère sensiblement de la doctrine libérale de "l'État de droit " et de la conception classique de la « souveraineté populaire ». Selon lui, le processus universel de "désenchantement du monde " vide de leur sens les doctrines libérales et démocratiques. Les " Droits de l'Homme " ne sont, à ses yeux, qu'un produit de "fanatismes rationalistes extrêmes". Même la vieille idée des "droits imprescriptibles de l'Homme » a perdu de sa force de conviction dans le contexte du capitalisme avancé, que caractérisent le règne de la bureaucratie et le pouvoir d'une oligarchie de chefs au service de " partis machines" et de puissances économiques. C'est pourquoi Mommsen souscrit au jugement de Georg Lukacz selon lequel la démocratie n'est pas autre chose pour Weber -

1. Jürgen KoCKA, « La bourgeoisie dans l'histoire moderne et contemporaine de l'Allemagne : recherche et débats récents ", Le Mouvement social, 136, 1986, p. 5-27.

2. Il s'agit en réalité de deux conférences de Max Weber : 1) "Politik als Beruf ";2) «Wissenschaft als Beruf " réunies dans la trad. franç. sous le titre "Le Savant et le Politique $"$, Paris, Plon, 1959. 
dont il fut le disciple contestataire - « qu'une mesure technique en faveur d'un meilleur fonctionnement de l'impérialisme " ${ }^{3}$.

Ce n'est donc pas un effet du hasard si Mommsen consacre le chapitre le plus long, soit plus d'un quart de son étude, à la conception wébérienne de l'impérialisme allemand. Sans partager l'ivresse guerrière de l'opinion allemande en 1914 à propos d'un conflit qu'il jugeait inéluctable dans la compétition entre grandes puissances, Weber oppose, à l'irréalisme des objectifs de guerre wilhelmiens, le réalisme de la politique expansionniste de Bismarck. Rejetant toute idée d'expansion à l'Ouest jugée suicidaire pour l'Allemagne parce qu'elle susciterait une hostilité permanente des puissances occidentales, Weber estime que la grande mission politique du Reich est à l'Est. Même s'il ne souscrit pas au vieux rêve de germanisation, repris par les pangermanistes dont il combat d'ailleurs les outrances, son projet vise à isoler la Russie par la " libération " des petits États d'Europe orientale de sa tutelle en leur accordant une autonomie assortie d'une union économique et douanière avec le Reich et l'Autriche-Hongrie. Projet proche pour l'essentiel de la Mitteleuropa que son fidèle disciple Friedrich Naurann, avec lequel il coopère d'ailleurs au sein de la Commission d'étude créée à cet effet, publie en 1915, même si Weber se montre plus réticent à l'égard d'un condominium germano-autrichien. La proclamation d'un royaume de Pologne, en novembre 1916, par Ludendorff pressé de mettre sur pied des divisions polonaises pour soulager l'armée allemande, et la Révolution d'octobre 1917 en Russie, dans laquelle Weber ne voit que le remplacement d'un souverain incompétent par "l'impérialisme populaire russe " ne modifient en rien cette conception de l'expansion à l'Est dont certains historiens de R.F.A. ont souligné la permanence dans la politique allemande du $x^{e}$ siècle $^{4}$. C'est aussi pour sauvegarder les chances ultérieures de cette politique, que Weber soutiendra, après l'avoir durement critiquée dans une série d'articles de la Frankfurter Zeitung à l'automne 1917, la résolution de paix adoptée par le Reichstag. À ce propos, Weber n'avait pas hésité à dénoncer « le rebut des peuples sauvages d'Asie et d'Afrique, accompagné de tous les brigands et toutes les canailles de la terre, prêts à dévaster l'Allemagne... ", ce que Mommsen réduit à un "dérapage vers les bas-fonds de la propagande guerrière " (p. 339) en écartant un peu trop rapidement, me semble-t-il, l'influence des thèses racistes et antisémites sur la bourgeoisie de l'époque, aussi bien à ce propos que lorsque Weber s'opposera, en août 1919, à ce que plus d'un tiers de personnalités d'origine juive siègent dans la Commission d'enquête parlementaire sur les responsabilités de la guerre afin de ne pas gêner l'audition d'officiers supérieurs allemands (p. 414). Les égards de Weber pour la caste militaire relèvent, il est vrai, en partie de considérations d'ordre tactique, tout comme son soutien à la social-démocratie qui lui paraît seule capable, au lendemain de la défaite, d'en finir avec le " carnaval sanglant " de "la bande à Liebknecht " dont les membres sont traités de "fous ", de

3. Georg Lukacz, Die Zerstörung der Vernunft, Berlin (DDR), Aufbau Verlag, 1954.

4. Cf. la bibliographie in Raymond PoIDEVIN, L'Allemagne et le monde au XXe siècle, Paris, Masson, 1983, p. 275-278. 
" canailles " et autres aménités du même acabit. Quant aux militaires, bien qu'il désapprouve la parodie de retour triomphal en 1918 et la démission des officiers supérieurs comme de leur chef suprême dans le contexte de la défaite, allant jusqu'à préconiser le remplacement de l'armée traditionnelle par une milice populaire, il n'aura de cesse, six mois plus tard et jusqu'à sa mort, de vouloir restaurer le prestige de la caste militaire, de plaider pour la reconstitution du Grand Etat-major, fût-ce en violation du Traité de Versailles. Il ira jusqu'à approuver l'action des corps francs comme solution transitoire en attendant l'action future de grande envergure à l'Est.

Le ralliement à la République relève, lui aussi, de la tactique. Contrairement à certains bourgeois libéraux comme Stresemann, qui s'adaptent trop hâtivement à son gré aux données nouvelles - dont le projet de la S.D.N. qu'il rejette catégoriquement - Weber va jusqu'à refuser de souscrire au document constitutif du Parti démocrate allemand (D.D.P.) dont il est pourtant l'un des fondateurs parce que celui-ci se déclare en faveur d'une forme d'Etat républicaine. Car il ne désespère pas de voir son pays revenir à une monarchie parlementaire à laquelle coopéreraient les sociaux-démocrates, comme ils l'avaient fait lors de l'éphémère gouvernement du prince de Bade en 1918. Même ses amis du Parti démocrate - dont il ne tarde pas à démissionner - ne comprennent pas ses volte-face, la contradiction entre son discours et sa pratique. Attitude qui s'explique pourtant par la distinction qu'il établit entre "Le Savant " et « le Politique $"^{5}$. D'où son impuissance, aussi manifeste sous l'Empire que sous la République, à jouer un rôle actif dans la politique même si certaines de ses idées ont fait leur chemin dans les sphères dirigeantes.

Reste, comme le souligne Mommsen, sa contribution importante à la rédaction de la Constitution de Weimar. Si le président Ebert dut renoncer, après ses diatribes antirévolutionnaires, à le nommer secrétaire d'État à l'Intérieur, chargé de la rédaction du projet de constitution, Hugo Preuss, démocrate indépendant désigné à sa place, le nomma à la commission d'experts à laquelle il participa activement. C'est à lui que revient le mérite d'un compromis entre la conception centralisatrice du libéralisme prussien et les thèses fédéralistes des États d'Allemagne du Sud. Il parvient aussi à faire admettre le droit d'enquête parlementaire qu'il n'avait cessé de réclamer sous l'Empire, afin d'endiguer l'omnipotence de la bureaucratie administrative. Mais sa revendication essentielle d'un président du Reich, chef autonome de l'Exécutif, directement élu par le peuple comme pivot du système politique et contrepoids à un Parlement médiatisé par les appareils politiques et corporatifs, n'est pas retenue dans la Constitution. Le Président sera certes élu au suffrage universel et disposera d'importantes prérogatives, notamment celle de gouverner par ordonnances en cas de crise ou de péril grave (art. 48). Sur ce point, Mommsen, contrairement à l'opinion la plus répandue parmi les spécialistes, ne considère pas cet article comme une cause essentielle du déclin du régime parlementaire de Weimar. Le

5. "Le politicien, écrit M. Weber dans une lettre à sa sæur, ne peut que faire des compromis. L'intellectuel ne doit pas les couvrir ", cité in Marianne WeBER, Max Weber, ein Lebensbild, lre éd. Tubingen, 2e éd. Heidelberg, 1950, p. 702. 
pouvoir d'un " monarque élu ", souhaité par Weber, lui serait venu progressivement de l'opinion publique et des hommes politiques. Ceux-ci tendaient à lui reconnaître, surtout après l'élection en 1925 du maréchal-président Hindenburg, les pouvoirs jadis dévolus à l'empereur, et à penser qu'il offrait une alternative politique à la crise, ce qui aurait accrédité l'idée d'un Sauveur providentiel.

L'argument de Mommsen ne manque pas de poids dans la mesure où il rappelle que le petit groupe de juristes bourgeois républicains pas plus que la social-démocratie allemande n'avaient réfléchi sérieusement aux modalités d'un régime républicain et démocratique. D'où la facilité avec laquelle le juriste Carl Schmitt, "Méphisto de l'ère préhitlérienne ", pousse la thèse wébérienne jusqu'à la logique extrême du chef charismatique tirant sa légitimité du plébiscite. Déduction dont on connaît la suite.

On suivra moins volontiers l'auteur dans ses réflexions à long terme, notamment sur «les idées, s'épanouissant en répercussions autonomes, parfois opposées aux motivations de leur créateur » (p. 485), qui rejoint d'ailleurs le débat actuel, déjà évoqué, sur "la voie particulière " ou non de l'Allemagne industrielle.

L'intérêt de l'étude de Mommsen est de révéler dans sa complexité l'itinéraire politique d'un représentant de cette bourgeoisie allemande plus prompte à chercher l'efficacité qu'à en mesurer les conséquences politiques. Peut-être W. Mommsen nous incite-t-il, même s'il invite à chercher d'autres voies, à mieux comprendre certains traits permanents jusque dans la bourgeoisie ouest-allemande d'aujourd'hui ? Ce n'est là bien sûr qu'une question parmi d'autres qui vient à l'esprit du lecteur de cet ouvrage dense et bien documenté, que complètent un index des personnes citées et une bibliographie de 27 pages dont on regrettera, comme le signale l'auteur lui-même, qu'elle soit arrêtée à la date de parution de la $2^{e}$ édition allemande (1974) mais que l'on pourra compléter grâce à une étude bibliographique récente de Jürgen Kocka et Detlev J. K. Peukert ${ }^{\circ}$. On aurait aussi aimé trouver un tableau chronologique des faits marquants de la vie de Weber, trop rapidement esquissés dans le premier chapitre.

Rita R. Thalmann.

6. Jürgen Kocka, Detlev J. K. Peukert, « Max Weber et l'histoire. Derniers développements en République fédérale d'Allemagne ", Revue de synthèse, IVe S., 1-2, janv.-juin 1986, p. 9-37. 